“(C) 2015 IEEE. Personal use of this material is permitted. Permission from IEEE must be obtained for all other uses, in any current or future media, including reprinting/republishing this material for advertising or promotional purposes, creating new collective works, for resale or redistribution to servers or lists, or reuse of any copyrighted component of this work in other works." 


\title{
Fast Global Scan Matching for High-Speed Vehicle Navigation
}

\author{
Tomonari Furukawa ${ }^{1,2}$, Lakshitha Dantanarayana ${ }^{2}$, Jason Ziglar $^{1}$, Ravindra Ranasinghe ${ }^{2}$ \\ and Gamini Dissanayake ${ }^{2}$
}

\begin{abstract}
This paper presents a fast global scan matching technique for high-speed vehicle navigation. The proposed grid-based scan-to-map matching technique collectively handles unprocessed scan points at each grid cell as a grid feature. The grid features are transformed and located in the global frame and updated every time a new scan is acquired. Since registered and updated are only grid features, which are each the mean of scan points in a grid cell, the proposed grid feature matching technique is very fast. Representation for each grid cell by multiple grid features further maintains accuracy regardless of the grid size while fast processing is achieved. The technique is therefore suited for localization of high-speed vehicle navigation. Experimental results show the effectiveness of the proposed technique numerically and experimentally.
\end{abstract}

\section{INTRODUCTION}

While mobile robots have been successfully navigated by Global Positioning Systems (GPSs) in known environments, navigation of mobile robots in unknown environments still remains challenging. The robots cannot be navigated by GPSs because of the unavailability of the paths to follow. This gives rise to need for Simultaneous Localization and Mapping (SLAM) and other registration-based localization and mapping techniques, which iteratively update the map of local environments and the location of the mobile robot. A series of waypoints can be introduced once the local map and the robot location has been known.

Since successful localization and mapping relies upon the successful association of the new observation to the past observations, existing techniques can be classified in terms of the type of data extracted from the observation into two categories, feature-based and scan-based. In the featurebased techniques, a set of features including different types of geometric models such as points, lines, curvatures, and any arbitrary shapes is extracted from the observation and used as landmarks to associate the new and previous observations [1], [2]. The advantage of the feature-based technique is fast computation since a limited number of features are to be processed. Its disadvantages are accuracy and reliability. Accuracy could be significantly dropped since only data representing extracted features are utilized for mapping and localization whilst the remaining are discarded. Reliability is an issue since feature extraction is challenging and may not be successful [3], [4], [5].

\footnotetext{
${ }^{1}$ Tomonari Furukawa and Jason Ziglar are primarily with the Department of Mechanical Engineering, Virginia Tech, Blacksburg, VA, USA \{tomonari,jpz\}@vt.edu

${ }^{2}$ All except Jason Ziglar are with the Center for $\mathrm{Au}-$ tonomous Systems, University of Technology, Sydney, NSW, Australia ttomonari.furukawa, lakshitha.dantanarayana, ravindra.ranasinghe, gamini.dissanayake\}@uts.edu.au
}

The scan-based techniques [6], [7], [8], [9], on the other hand, directly utilizes unprocessed scanned data as observations which are generally sets of points obtained from range sensors such as a sonar and a laser scanner. One of the fundamental challenges of the scan-based techniques, as an approach to associate the new scan to the past scans, has thus been the association or matching of one scan to another, namely the scan-to-scan matching. The most common scanto-scan matching approach is based on the Iterative Closest Point (ICP) technique [10] which allows the point-to-point matching between two scans by minimizing the total distance between them. Despite the popularity of the technique, the point-to-point matching may yield inappropriate data association since the matching is dominated by the densely populated neighboring points. Weiss and Puttkamer [11] proposed an improved technique that additionally considers an angle between two neighboring scan points. Biber and Straßer [12] introduced a grid space and matched scans by collectively representing scan point in each grid as a normal distribution in the name of Normal Distribution Transform (NDT). Although they could exhibit improved accuracy due to the full use of unprocessed data, these local pointto- $X$ techniques inevitably yield and accumulate the scan matching error while making the process computationally expensive unless accuracy is intensionally dropped as a tradeoff.

This paper presents a fast global scan matching technique, which has been proposed by taking the advantages of both the feature-based and scan-based techniques. The proposed technique handles unprocessed scan points similarly to the scan-based techniques but collectively represents scan points as features by introducing a grid to the space of concern and features of each grid cell, or grid features (GFs). A global grid is defined with the initial robot pose as the reference, and all the scans are registered onto the global grid. In the registration, GFs of each scan are matched to the map GFs, which are already registered in the global grid, in the name of grid-based scan-to-map matching. One of the features of this technique is its ability to control efficiency and accuracy rather independently since the technique can locate multiple map GFs in each grid cell. While efficiency depends on the grid size, the accuracy is most influenced not by the grid size but by the location and number of map GFs. The proposed technique can thus be both efficient and accurate by using a large grid size. The accuracy of the proposed technique can further be improved as scans are fully utilized to derive GFs.

This paper is organized as follows. The next section explains the general framework of the global scan matching 
framework whereas the proposed grid-based technique for fast vehicle navigation is presented in Section III. Section IV presents experimental results which investigate the effects of the proposed technique whereas conclusions and future work are summarized in the final section.

\section{Global SCAN MATCHING}

Figure 1 shows the overall process of the global scan matching, which is also called the scan-to-map matching. Unlike the conventional scan matching, or the scan-toscan matching, where the new scan is matched only to the previous scan, the global scan matching matches the new scan to all the past scans that are registered onto the the globally defined map. To understand the overall process mathematically, let the previous scan in the previous robot frame be $\{R-\} Z_{k-1}=\left\{\{R-\} \mathbf{z}_{k-1}^{i} \mid \forall i \in\{1, \cdots, m\}\right\}$ and the new scan in the new robot frame be ${ }^{\{\mathrm{R}\}} Z_{k}=$ $\left\{{ }^{\{R\}} \mathbf{z}_{k}^{i} \mid \forall i \in\{1, \cdots, m\}\right\}$ where $k$ is the time step and $m$ is the number of points in the scan. $\{R-\}$ and $\{R\}$ denote the robot frame at time step $k-1$ and the that at time step $k$.

When the new scan ${ }^{\{R\}} Z_{k}$ is obtained, the global scan matching first estimates the transformation matrix $\{R-\} \mathbf{p}_{k}^{s}$ using laser scanner readings together with any scan-to-scan matching technique such as ICP and NDT, the encoders readings, the Inertial Measurement Unit (IMU) readings, or their combination. Out of them, the scan-to-scan matching with laser readings is most powerful and effective since the laser reading is less subject to noise. It is generically given by the optimization problem

$$
\sum_{i=1}^{m} d\left(\{R-\} \mathbf{z}_{k}^{i}\left(\begin{array}{l}
\{R-\} \\
\{R\}
\end{array} \mathbf{p}_{k}^{s}\right),{ }^{\{\mathrm{R}-\}} \mathbf{Z}_{k}^{i}\right) \rightarrow \min _{\substack{\{R-\} \\
\{R\}}} \mathbf{p}_{k}^{s}
$$

where $d(\cdot)$ is a distance measure to minimize, and ${ }^{\{\mathrm{R}-\}} \mathbf{Z}_{k}^{i}$ is corresponding information constructed from the previous scan. The powerfulness and effectiveness are however true only when the laser scanner observes surroundings in close proximity and the robot movement, particularly the orientation change, is small. The optimization could otherwise fail since the ill-posed objective function in Eq. (1) could be multi-modal. The encoders and the IMU are used to make the objective function better-posed and provide a better initial guess to the optimization problem. After the derivation of $\{R-\}$
$\{R\}$ $\mathbf{p}_{k}^{s}$, each new scan point in the $\{R\}$ frame is then transformed to that in the $\{R-\}$ frame:

$$
{ }^{\{R-\}} \mathbf{z}_{k}^{i}\left(\begin{array}{l}
\{R-\} \\
\{R\}
\end{array} \mathbf{p}_{k}^{s}\right)=\mathbf{R}\left(\phi_{k}^{s}\right){ }^{\{R\}} \mathbf{z}_{k}^{i}+\mathbf{t}_{k}^{s}
$$

In order for global correction, each new scan point ${ }^{\{R-\}} \mathbf{z}_{k}^{i}$ is further transformed to that in the global frame $\{G\}$ using the robot pose estimated at the previous time step in the $\{G\}$ frame:

$$
{ }^{\{G\}} \mathbf{z}_{k}^{i}=\mathbf{R}\left({ }^{\{G\}} \theta_{k-1}\right)^{\{R-\}} \mathbf{z}_{k}^{i}+{ }^{\{G\}} \mathbf{x}_{k-1}
$$

where ${ }^{\{G\}} \mathbf{x}_{k-1}=\left[{ }^{\{G\}} x_{k-1},{ }^{\{G\}} y_{k-1}\right]^{\top}$ and ${ }^{\{G\}} \theta_{k-1}$ are the robot pose in the global frame estimated at time step $k-1$.
The iterative estimation of the robot pose in the global frame is performed by considering the robot movement, ${ }^{\{R-\}} \mathbf{X}_{k}$ and ${ }^{\{R-\}} \theta_{k}$, which is equivalent to $\mathbf{t}_{k}^{s}$ and $\phi_{k}^{s}$, respectively:

$$
\begin{aligned}
{ }^{\{G\}} \mathbf{x}_{k} & =\mathbf{R}\left(\{G\} \theta_{k-1}\right) \mathbf{t}_{k}^{s}+\{G\} \mathbf{x}_{k-1} \\
{ }^{\{G\}} \theta_{k} & =\phi_{k}^{s}+\{G\} \theta_{k-1}
\end{aligned}
$$

where the initial pose of the robot, $\left[\mathbf{x}_{0}, \theta_{0}\right]$, defines the global frame.

The new scan and the robot pose may, however, be incorrectly located due to the scan matching error, and the error accumulates over time since the scan matching is only local. Once the new scan is transformed to the $\{G\}$ frame, the global scan matching further matches the new scan to the map in the $\{G+\}$ frame, which is the global frame corrected by the global scan matching from the original guess of the global frame. The optimization problem is given by

$$
\sum_{i=1}^{m} d\left({ }^{\{G+\}} \mathbf{z}_{k}^{i}\left(\begin{array}{l}
\{G+\} \\
\{G\}
\end{array} \mathbf{p}_{k}^{m}\right),{ }^{\{\mathrm{G}+\}} \mathbf{Z}_{k}^{i}\right) \rightarrow \min _{\substack{\{G+\} \\
\{G\}}} \mathbf{p}_{k}^{m}
$$

where ${ }_{\{G\}}^{\{G+\}} \mathbf{p}_{k}^{m}=\left[\mathbf{t}_{k}^{m} \boldsymbol{\top}, \phi_{k}^{m}\right]^{\top}$ is the global scan matching transformation matrix, and transforms the new scan to the corrected global frame. The derivation of the global scan matching transformation matrix is detailed in the next subsections. Following the identification of $\{G+\} \mathbf{p}_{k}^{m}$, the new scan in the $\{G+\}$ frame is found as

$$
\{G+\} \mathbf{z}_{k}^{i}\left(\begin{array}{l}
\{G+\} \\
\{G\}
\end{array} \mathbf{p}_{k}^{m}\right)=\mathbf{R}\left(\phi_{k}^{m}\right){ }^{\{G\}} \mathbf{z}_{k}^{i}+\mathbf{t}_{k}^{m}
$$

Simultaneously, the robot pose in the $\{G\}$ frame is also corrected by $\{G+\} \mathbf{p}_{k}^{m}$ :

$$
\begin{aligned}
\{G+\} \mathbf{x}_{k} & =\mathbf{R}\left(\phi_{k}^{m}\right)^{\{G\}} \mathbf{x}_{k}+\mathbf{t}_{k}^{m} \\
\{G+\} \theta_{k} & ={ }^{\{G\}} \theta_{k}+\phi_{k}^{m}
\end{aligned}
$$

Because the scan matching error is removed, the global scan matching does not accumulate the error.

\section{GRID-BASED SCAN-TO-MAP MATCHING}

\section{A. Grid Representation and Scan Grid Features}

Once it is acquired and converted to the $\{G\}$ frame, the new scan is ready to be represented on a grid as a set of GFs. Let the grid cells containing scan points more than the minimum number of scan points be

$$
S_{k}=\left\{\{G\}_{\overline{\mathbf{z}}_{k}^{j}} \mid \forall j \in\left\{1, \cdots, n_{k}^{s}\right\}\right\}
$$

where $n_{k}^{s}$ is the number of grid cells containing scan points more than the minimum number $m_{\min }^{s}$. The scan GF in the $j$ th grid cell is derived simply as

$$
\{G\}_{\overline{\mathbf{z}}_{k}^{j}}=\frac{1}{m_{k}^{s_{j}}} \sum_{i=1}^{m_{k}^{s_{j}}}\{G\}_{\mathbf{z}_{k}^{j_{i}}}
$$

where $m_{k}^{s_{j}}$ is the number of scan points in the $j$ th cell, and ${ }^{\{G\}} \mathbf{z}_{k}^{j_{i}}$ is the $i$ th scan point in the $j$ th cell. 


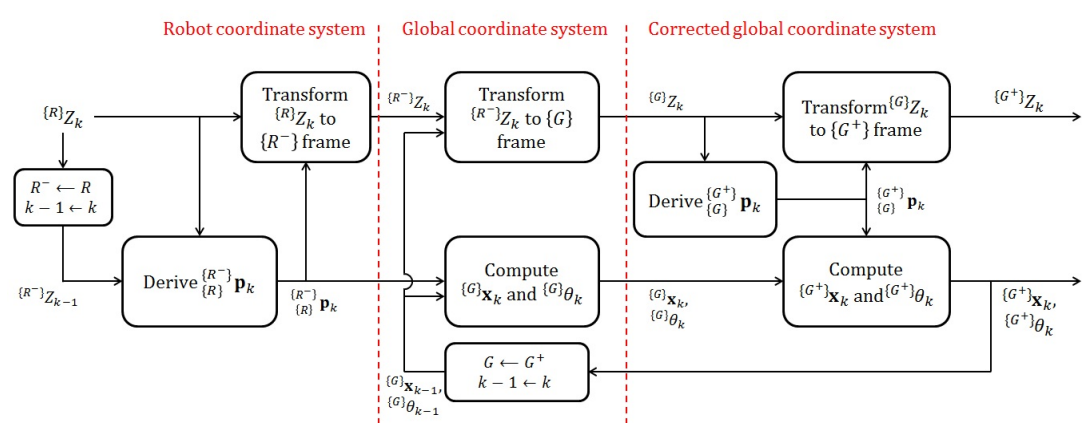

Fig. 1. Overall process of global scan matching

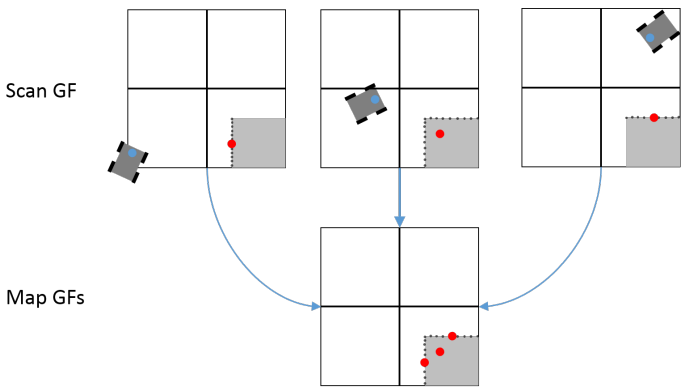

Fig. 2. Grid-based scan-to-map matching

\section{B. Map Grid Features and Selection of Corresponding Map Grid Feature}

Figure 2 illustratively shows the motivation of representing and handling multiple map GFs in each grid cell. As shown in the figure, the scan GF of an object can be significantly different depending on the robot pose. The map with multiple GFs allows the matching of the new scan to a map GF irrespective of the robot pose. Mathematically, grid cells registering at least one scan GF up to time step $k-1$ are represented as

$$
M_{1: k-1}=\left\{M_{1: k-1}^{j} \mid \forall j \in\left\{1, \cdots, n_{k-1}^{g}\right\}\right\}
$$

where $M_{1: k-1}^{j}$ is the property of the $j$ th grid cell, and $n_{k-1}^{g}$ is the number of grid cells. $M_{1: k-1}^{j}$ is given by

$$
\begin{array}{r}
M_{1: k-1}^{j}=\left\{M_{1: k-1}^{j_{l}}=\left\{\overline{\mathbf{z}}_{1: k-1}^{j_{l}}, m_{k-1}^{j_{l}}\right\}\right. \\
\left.\mid \forall l \in\left\{1, \cdots, n_{k-1}^{g_{j}}\right\}\right\}
\end{array}
$$

where $M_{1: k-1}^{j_{l}}$ is the property of the $l$ th map GF in the $j$ th cell with the mean, $\overline{\mathbf{z}}_{1: k-1}^{j_{l}}$, and the total number of scan points, $m_{k-1}^{j_{l}} \cdot n_{k}^{g_{j}}$ denotes the total number of map GFs in the $j$ th cell, and a corresponding map GF must be selected if $n_{k}^{g_{j}} \geq 2$.

The selection of a corresponding map GF for the scan GF in the proposed technique starts with quantifying the distance of the scan GF to each map GF in the same cell and finding the closest map GF:

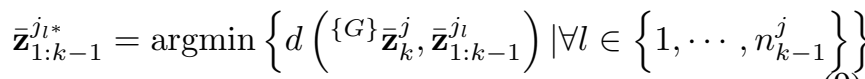

where

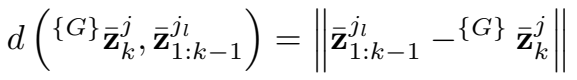

If the distance is close enough, or less than the threshold, the closest map GF should become the corresponding map GF. If not, a new map GF should be created since no map GF is similar. The selection of the corresponsing map GF is resultantly given by

$$
\hat{\mathbf{z}}_{k}^{j}= \begin{cases}\overline{\mathbf{z}}_{1: k-1}^{j_{l}} & \text { if } \operatorname{argmin}\{d(\cdot)\}<\gamma \\ \{G\}_{\mathbf{z}_{k}^{j}}^{j} & \text { Otherwise }\end{cases}
$$

\section{Identification of Error Correction Parameters}

Having the matching map GF identified for each scan GF, the transformation matrix ${ }_{\{G\}}^{\{G+\}} \mathbf{p}_{k}^{m}$ can be identified by matching all the scan GFs to the corresponding matching map GFs. The identification of $\{G+\} \mathbf{p}_{k}^{m}$ begins with the initial values set to $\mathbf{0}$ as it is valid to assume that ${ }_{\{R\}}^{\{R-\}} \mathbf{p}_{k}^{s}$ and the previous robot pose estimation is reasonably correct. The proposed technique first transforms each scan GF to that in the $\{G+\}$ frame using the currently guessed ${ }_{\{G\}}^{\{G+\}} \mathbf{p}_{k}^{m}$ :

$$
{ }^{\{G+\}} \overline{\mathbf{z}}_{k}^{j}\left(\begin{array}{l}
\{G+\} \\
\{G\}
\end{array} \mathbf{p}_{k}^{m}\right)=\mathbf{R}\left(\phi_{k}^{m}\right)^{\{G\}} \overline{\mathbf{z}}_{k}^{j}+\mathbf{t}_{k}^{m}
$$

With all the scan GFs and the matching map GFs described in the $\{G+\}$ frame, the transformation matrix ${ }_{\{G\}}^{\{G+\}} \mathbf{p}_{k}^{m}$ can be then computed by minimizing the objective function given by the sum of differences between the scan GFs and the corresponding matching map GFs:

$$
\Phi\left(\begin{array}{l}
\{G+\} \\
\{G\}
\end{array} \mathbf{p}_{k}^{m}\right)=\sum_{j=1}^{n_{k}^{s}} D\left(\{G+\} \overline{\mathbf{z}}_{k}^{j}, \hat{\mathbf{z}}_{k}^{j}\right) \rightarrow \min _{\substack{\{G+\} \\
\{G\}}} \mathbf{p}_{k}^{m}
$$

where

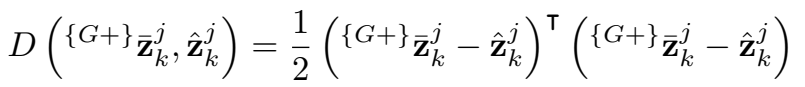

The objective function of the proposed technique allows the global scan matching with improved accuracy by matching to multiple GFs while requiring the transformation and matching of only GFs instead of all the scan points. This makes the proposed technique efficient and accurate.

Applying Newton-Raphson method, $\{G+\} \mathbf{p}_{k}^{m}$ is iteratively computed by the increment $\Delta_{\{G\}}^{\{G+\}} \mathbf{p}_{k}^{m}$ :

$$
\Delta_{\{G\}}^{\{G+\}} \mathbf{p}_{k}^{m}=-\mathbf{H}_{k}^{-1} \mathbf{g}_{k}
$$


where $\mathbf{H}_{k}$ and $\mathbf{g}_{k}$ are the sums of the Hessian, $\tilde{\mathbf{H}}_{k}$, and the gradient, $\tilde{\mathrm{g}}_{k}$, of the score function:

$$
\mathbf{H}_{k}=\sum_{j=1}^{n_{k}^{s}} \tilde{\mathbf{H}}_{k}^{j}, \quad \mathbf{g}_{k}=\sum_{j=1}^{n_{k}^{s}} \tilde{\mathbf{g}}_{k}^{j}
$$

For the $j$ th cell, the gradient vector $\tilde{\mathbf{g}}_{k}^{j}$ is given by

$$
\tilde{\mathbf{g}}_{k}^{j}=\frac{\partial^{\{G+\}} \overline{\mathbf{z}}_{k}^{j}}{\partial_{\{G\}}^{\{G+\}} \mathbf{p}_{k}^{m}}\left({ }^{\{G+\}} \overline{\mathbf{z}}_{k}^{j}-\hat{\mathbf{z}}_{k}^{j}\right)
$$

and the Hessian matrix $\tilde{\mathbf{H}}_{k}^{j}$ is computed by

$$
\begin{gathered}
\tilde{\mathbf{H}}_{k}^{j}=\frac{\partial^{\{G+\}} \overline{\mathbf{z}}_{k}^{j}}{\partial_{\{G\}}^{\{G+\}} \mathbf{p}_{k}^{m}} \frac{\partial^{\{G+\}_{\mathbf{z}_{k}^{j}}}{ }^{\top}}{\partial_{\{G\}}^{\{G+\}} \mathbf{p}_{k}^{m}} \\
+\frac{\partial^{2\{G+\}} \overline{\mathbf{z}}_{k}^{j}}{\partial_{\{G\}}^{\{G+\}} \mathbf{p}_{k}^{m}}\left({ }^{2}{ }^{2 G+\}} \overline{\mathbf{z}}_{k}^{j}-\hat{\mathbf{z}}_{k}^{j}\right)
\end{gathered}
$$

where

$$
\frac{\partial^{\{G+\}} \overline{\mathbf{z}}_{k}^{j}}{\partial_{\{G\}}^{\{G+\}} \mathbf{p}_{k}^{m}}=\nabla\left(\mathbf{R}\left(\phi_{k}^{m}\right)^{\{G\}} \overline{\mathbf{z}}_{k}^{j}+\mathbf{t}_{k}^{m}-\hat{\mathbf{z}}_{k}^{j}\right)
$$

and

$$
\frac{\partial^{2\{G+\}} \overline{\mathbf{z}}_{k}^{j}}{\partial_{\{G\}}^{\{G+\}} \mathbf{p}_{k}^{m^{2}}}=\frac{\partial}{\partial_{\{G\}}^{\{G+\}} \mathbf{p}_{k}^{m}}\left(\frac{\partial^{\{G+\}} \overline{\mathbf{z}}_{k}^{j}}{\partial_{\{G\}}^{\{G+\}} \mathbf{p}_{k}^{m}}\right)
$$

If this is two-dimensional, GFs in the $\{G+\}$ frame are

$$
\begin{array}{r}
\{G+\}_{\mathbf{z}_{k}^{j}}\left(\begin{array}{l}
\{G+\} \\
\{G\}
\end{array} \mathbf{p}_{k}^{m}\right)=\mathbf{R}\left(\phi_{k}^{m}\right)^{\{G\}} \overline{\mathbf{z}}_{k}^{j}+\mathbf{t}_{k}^{m} \\
=\left[\begin{array}{cc}
\cos \phi_{k}^{m} & -\sin \phi_{k}^{m} \\
\sin \phi_{k}^{m} & \cos \phi_{k}^{m}
\end{array}\right]\left[\begin{array}{c}
\{G\} z_{k}^{j_{x}} \\
\{G\} z_{k}^{j_{y}}
\end{array}\right]+\left[\begin{array}{c}
t_{k}^{m_{x}} \\
t_{k}^{m}
\end{array}\right] \\
=\left[\begin{array}{c}
\{G\} z_{k}^{j_{x}} \cos \phi_{k}^{m}-\{G\} z_{k}^{j_{y}} \sin \phi_{k}^{m}+t_{k}^{m_{x}} \\
\{G\} z_{k}^{j_{x}} \sin \phi_{k}^{m}+\{G\} z_{k}^{j_{y}} \cos \phi_{k}^{m}+t_{k}^{m_{y}}
\end{array}\right]
\end{array}
$$

The derivative in Equation (17) is given by

$$
\frac{\partial^{\{G+\}} \overline{\mathbf{z}}_{k}^{j}}{\partial_{\{G\}}^{\{G+\}} \mathbf{p}_{k}^{m}}=\nabla\left(\mathbf{R}\left(\phi_{k}^{m}\right)^{\{G\}} \overline{\mathbf{z}}_{k}^{j}+\mathbf{t}_{k}^{m}-\hat{\mathbf{z}}_{k}^{j}\right)=\left[\begin{array}{cc}
1 & 0 \\
0 & 1 \\
a & b
\end{array}\right]
$$

where

$$
\begin{gathered}
a=-{ }^{\{G\}} z_{k}^{j_{x}} \sin \phi_{k}^{m}-{ }^{\{G\}} z_{k}^{j_{y}} \cos \phi_{k}^{m} \\
b={ }^{\{G\}} z_{k}^{j_{x}} \cos \phi_{k}^{m}-{ }^{\{G\}} z_{k}^{j_{y}} \sin \phi_{k}^{m}
\end{gathered}
$$

The gradient is thus derived as

$$
\mathbf{g}_{k}=\left[\begin{array}{c}
z_{k}^{j_{x}}-\bar{z}_{k}^{j_{x}} \\
z_{k}^{j_{y}}-\bar{z}_{k}^{j_{y}} \\
a\left(z_{k}^{j_{x}}-\bar{z}_{k}^{j_{x}}\right)+b\left(z_{k}^{j_{y}}-\bar{z}_{k}^{j_{y}}\right)
\end{array}\right]
$$

By further differentiating the gradient, Hessian matrix can be derived:

$$
\frac{\partial}{\partial t_{k}^{m_{x}}}\left(\frac{\partial^{\{G+\}_{\mathbf{z}_{k}^{j}}}}{\partial_{\{G\}}^{\{G+\}} \mathbf{p}_{k}^{m}}\right)=\left[\begin{array}{cc}
0 & 0 \\
0 & 0 \\
0 & 0
\end{array}\right]
$$

$$
\begin{gathered}
\frac{\partial}{\partial t_{k}^{m_{y}}}\left(\frac{\partial^{\{G+\}_{\mathbf{z}_{k}^{j}}}}{\partial_{\{G\}}^{\{G+\}} \mathbf{p}_{k}^{m}}\right)=\left[\begin{array}{ll}
0 & 0 \\
0 & 0 \\
0 & 0
\end{array}\right] \\
\frac{\partial}{\partial \phi_{k}^{m}}\left(\frac{\partial^{\{G+\}_{\mathbf{z}_{k}^{j}}}}{\partial_{\{G\}}^{\{G+\}} \mathbf{p}_{k}^{m}}\right)=\left[\begin{array}{ll}
0 & 0 \\
0 & 0 \\
c & d
\end{array}\right]
\end{gathered}
$$

where

$$
\begin{aligned}
& c=-{ }^{\{G\}} z_{k}^{j_{x}} \cos \left(\phi_{k}^{m}\right)+{ }^{\{G\}} z_{k}^{j_{y}} \sin \left(\phi_{k}^{m}\right) \\
& d=-{ }^{\{G\}} z_{k}^{j_{x}} \sin \left(\phi_{k}^{m}\right)-{ }^{\{G\}} z_{k}^{j_{y}} \cos \left(\phi_{k}^{m}\right)
\end{aligned}
$$

The substitution into Equation (18) yields the Hessian matrix. When $\Delta_{\{G\}}^{\{G+\}} \mathbf{p}_{k}^{m}$ is computed, $\{G\}{ }_{\{G\}} \mathbf{p}_{k}^{m}$ is then updated by:

$$
{ }_{\{G\}}^{\{G+\}} \mathbf{p}_{k}^{m} \leftarrow{ }_{\{G\}}^{\{G+\}} \mathbf{p}_{k}^{m}+\Delta_{\{G\}}^{\{G+\}} \mathbf{p}_{k}^{m}
$$

\section{Update of robot pose and map GFs}

The robot pose and the map GFs can be updated once the global transformation matrix has been determined. The robot pose is updated using Equation (7). The map GFs are updated differently depending on whether there is a corresponding

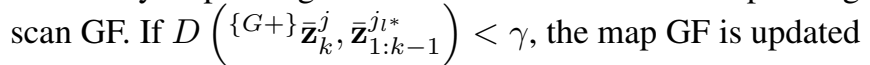
according to the weighted mean formulation:

$$
\begin{gathered}
\overline{\mathbf{z}}_{1: k}^{j_{1 *}}=\frac{m_{1: k-1}^{j} \overline{\mathbf{z}}_{1: k-1}^{j_{l *}}+m_{k}^{j}\{G+\}_{\mathbf{z}_{k}^{j}}^{j}}{m_{1: k-1}^{j}+m_{k}^{j}} \\
m_{1: k}^{j_{l *}}=m_{1: k-1}^{j_{j^{*}}}+m_{k}^{j}
\end{gathered}
$$

Otherwise, the scan GF becomes the new map GF:

$$
\begin{aligned}
& \overline{\mathbf{z}}_{1: k}^{j_{l *}}={ }^{\{G\}} \overline{\mathbf{z}}_{k}^{j} \\
& m_{k-1: k}^{j_{l *}}=m_{k}^{j}
\end{aligned}
$$

\section{EXPERIMENTAL RESUlts}

In order to investigate its performance, the proposed global scan matching technique was applied to the localization and mapping by a car with a drive-by-wire system. Cars require fast navigation while they may be operated in GPSdenied environments, so they are vehicles that would need the proposed technique. Figure 3(a) shows a parking area where the performance tests of the proposed technique were conducted whereas the car used for the tests is shown in Figure 3(b). The car is Toyota Prius and equipped with two laser scanners, Hokuyo UXM-30LX-EW; one at the front side of the car and the other at the rear side. Only the front laser scanner was used in the tests. In order to show the applicability of the proposed technique, the speed of the car was intentionally changed from nearly $0 \mathrm{~km} / \mathrm{h}$ up to $40 \mathrm{~km} / \mathrm{h}$. The car made a loop so that the loop closure error can be recognized and quantified. 


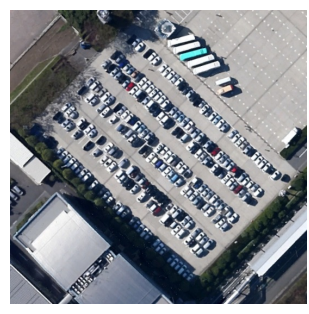

(a) Test environments

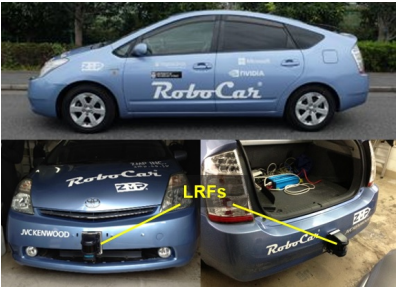

(b) Test vehicle
Fig. 3. Vehicle navigation test

TABLE I

PARAMETERS FOR THE PROPOSED GLOBAL SCAN MATCHING TECHNIQUE

\begin{tabular}{cl}
\hline Parameter & Value \\
\hline Grid size $[\mathrm{m}]$ & 1 \\
Threshold $\gamma$ & 1 \\
Minimum number of scan points $m_{\min }^{s}$ & 3 \\
\hline
\end{tabular}

\section{A. Map accuracy by the proposed technique and comparison to GMapping}

Table I lists parameters used in the proposed technique. As shown in the table, one of the advantage of this technique is the small number of parameters that governs the performance of the proposed technique. If the performance of the proposed technique is less sensitive to the grid size, only two parameters govern the performance.

Figure 4 shows the resulting map of the proposed technique, which is compared to that of the well-known GMapping. While blue circles are registered map GFs, the pink dots show the last scan indicating that the robot has made a loop. The proposed technique was able to develop a map with very little loop closure error whilst the GMapping has shown a significant amount of error without being able to close the loop. Because of the heavy computation of the particle filter based SLAM, the GMapping updated the map at a frequency of $1 \mathrm{~Hz}$. Change in orientation with this frequency is so large that the GMapping could not update well. On the other hand, the MATLAB based program of the proposed technique has been able to update a map at a frequency of $25 \mathrm{~Hz}$. The proposed technique could clearly construct a more accurate map by utilizing more consecutive scans with smaller changes in orientation. Figure 4(c) shows the zoomed view of the loop-closing area mapped by the proposed technique where red pluses and red circles are scan GFs and corresponding map GFs. It is seen that there is one map GF in a grid cell since $\gamma=1$.

\section{B. Effect of multiple map GFs}

Having the accuracy of the proposed technique validated, the effect of deploying multiple map GFs was investigated. Table II lists the parameters used in this test. The grid size was made large $(4 \mathrm{~m})$ as the resolution would be too large to

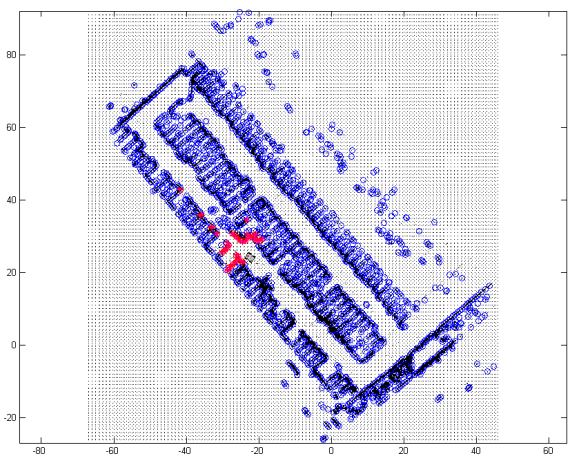

(a) Proposed technique

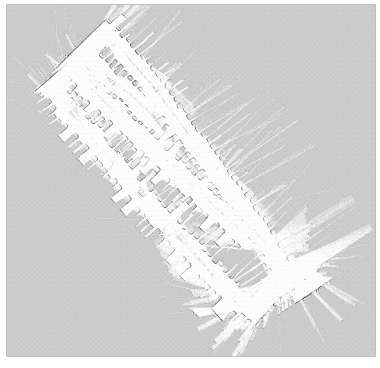

(b) GMapping

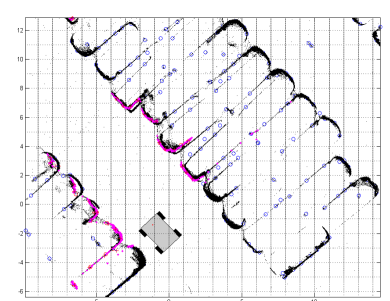

(c) Zoomed view
Fig. 4. Resulting map by the proposed technique and GMapping

TABLE II

PARAMETERS FOR INVESTIGATING EFFECT OF MULTIPLE MAP GFS

\begin{tabular}{cl}
\hline Parameter & Value \\
\hline Grid size $[\mathrm{m}]$ & 4 \\
Threshold $\gamma$ & {$[1,2,3,4]$} \\
Minimum number of scan points $m_{\min }^{s}$ & 3 \\
\hline
\end{tabular}

build a map with a single map GF. The threshold was thus changed from 4 to 1 to see the effect of multiple map GFs.

Figure 5 shows the map built with $\gamma=4$ whereas Figures 6 and 7 show those with $\gamma=2$ and $\gamma=1$, respectively. The map with $\gamma=4$ shows a heavily distorted map, which has resulted in the map as bad as that of the GMapping. This is because the threshold of $\gamma=4$ allows only one map GF for each grid cell, and the number of map GFs created is thus not large enough to achieve global scan matching. The maps with smaller thresholds $(\gamma=2$ and $\gamma=1$ ), on the other hand, allows multiple map GFs as shown in Figures 6(b) and 7(b) and makes the map more accurate.

\section{CONCLUSions AND Future Work}

In this paper, a fast global scan matching technique, which has been proposed by taking the advantages of both the feature-based and scan-based techniques, has been presented. The proposed technique handles unprocessed scan points 


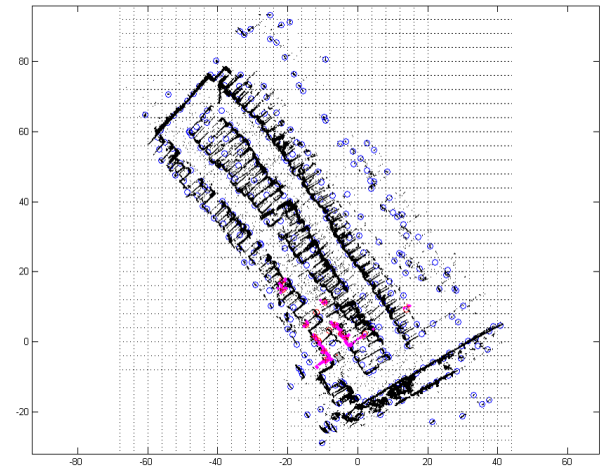

Fig. 5. Entire map with $\gamma=4$

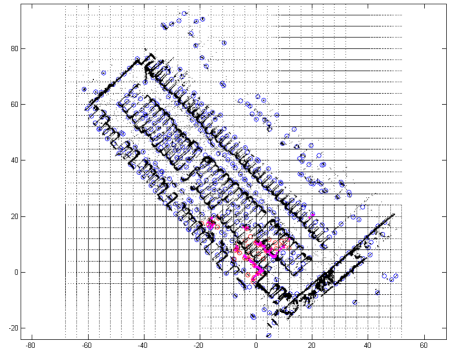

(a) Entire map

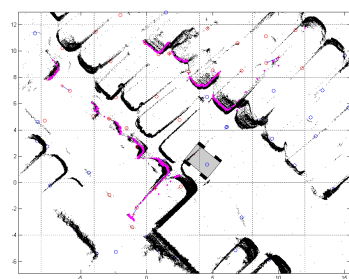

(b) Zoomed view
Fig. 6. Resulting map with $\gamma=2$

and collectively represents scan points as GFs on a globally defined grid. In the registration of scans, GFs of each scan are matched to the map GFs. One of the features of this technique is its ability to control efficiency and accuracy rather independently since the technique can locate multiple map GFs in each grid cell. The proposed technique can thus be both efficient and accurate by using a large grid size. The accuracy of the proposed technique can further be improved as scans are fully utilized to derive GFs unlike feature-based techniques that discard data unassociated with features.

The proposed technique was applied to the localization and mapping by a car with a drive-by-wire system. Results first show that the proposed technique can achieve accuracy superior to GMapping in part because it is fast and can utilize full scans without loss of information. The maintenance of high accuracy with a large grid size was also demonstrated, indicating the efficacy of the proposed technique.

The work presented in the paper is the first step, and various challenges are left open. Ongoing work includes the active loop closure, inexpensive data registration and incorporation of cloud computing, all of which are necessary for fast vehicle navigation.

\section{REFERENCES}

[1] Smith, R., and Cheeseman, P., 1987. "On the representation and estimation of spatial uncertainty". International Journal of Robotics Research, 5(4), pp. 56-68.

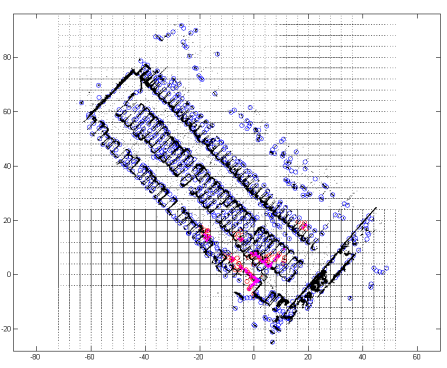

(a) Entire map

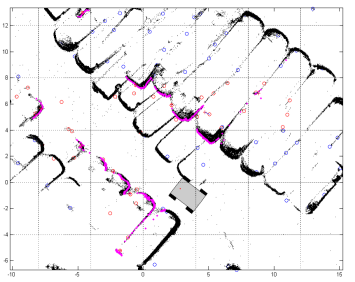

(b) Zoomed view
Fig. 7. Resulting map with $\gamma=1$

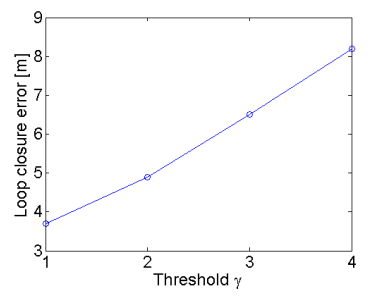

Fig. 8. Loop closure error vs $\gamma$

[2] Dissanayake, M.W.M.G. Newman, P. C. S. D.-W. H., and Csorb, M., 2001. "A solution to the simultaneous localization and map building (slam) problem". IEEE Transactions on Robotics and Automation, 17(3), pp. 229-241.

[3] Bar-Shalom, T., and Fortmann, T., 1988. Tracking and Data Association. Academic Press, Boston, MA.

[4] Feder, H.J.S., L. J., and Smith, C., 1999. "Adaptive mobile robot navigation and mapping". International Journal of Robotics Research, 18(7), pp. 650-668.

[5] Newman, P., 1999. On The Structure and Solution of the Simultaneous Localisation and Map Building Problem. Ph.D. thesis, Dept. Mechanical and Mechatronic Engineering, University of Sydney.

[6] Feng Lu, E. M., 1997. "Robot pose estimation in unknown environments by matching $2 \mathrm{~d}$ range scans". Journal of Intelligent and Robotic Systems, pp. 249-275.

[7] Diosi, A., and Kleeman, L., 2005. "Laser scan matching in polar coordinates with application to slam". In Proceedings of the IEEE/RSJ International Conference on Intelligent Robots and Systems, pp. 3317 3322

[8] A. Nüchter, J. Elseberg, P. S. D. P., 2010. "Linearization of rotations for globally consistent n-scan matching". In 2010 IEEE International Conference on Robotics and Automation, pp. 1373-1379.

[9] Olson, C., and Matthies, L., 1998. "Maximum likelihood rover localization by matching range maps". In Proceedings of the 1998 IEEE International Conference on Robotics and Automation, pp. 272 277.

[10] Besl, P. J., and McKay, N. D., 1992. "A method for registration of 3-d shapes". IEEE Transaction on Pattern Analysis and Machine Intelligence, 14(2).

[11] Weiss, G., and von Puttkamer, E., 1995. "A map based on laserscans without geometric interpretation". In Proceedings of Intelligent Autonomous Systems 4(IAS-4), pp. 403-407.

[12] Biber, P., and Strasser, W., 2003. "The normal distributions transform: A new approach to laser scan matching". In IEEE Int. Conf. on Intelligent Robots and Systems. 